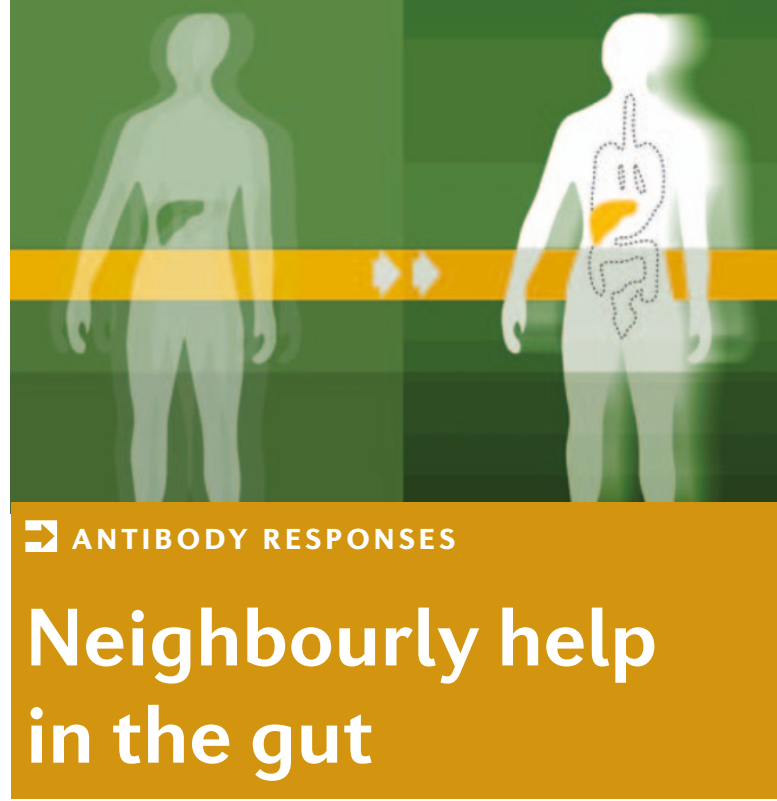

Most of the $\lg A$-secreting cells in the body are located in the intestine, and it is thought that the induction of class switching and targeting of these cells to the gut depends on signals from the gut-associated lymphoid tissue (GALT). But what are the signals that make a B cell home to the gut and specifically produce lgA? Mora et al. now show that the vitamin A metabolite retinoic acid produced by GALT-derived dendritic cells (DCs) confers gut tropism and, together with interleukin-6 (IL-6) or IL-5, induces IgA secretion.

DCs in the GALT, unlike DCs found in other lymphoid tissues, synthesize retinoic acid, which has been shown to induce the expression of a distinct set of trafficking molecules on the surface of T cells that infiltrate the gut. So, the authors examined the effect of GALT-derived DCs on expression of the gut-specific trafficking molecules $\alpha_{4} \beta_{7}$-integrin and CC-chemokine receptor 9 (CCR9) on B cells. Co-culture of $B$ cells with DCs derived from Peyer's patches resulted in $\alpha_{4} \beta_{7}$-integrin ${ }^{\text {hi }} C C R 9^{\text {hi }} B$ cells. By contrast, co-culture of $B$ cells with DCs isolated from peripheral lymph nodes induced $\alpha_{4} \beta_{7}$-integrin ${ }^{\text {low }} C C R 9^{\text {low }} B$ cells. Interestingly, expression of these homing molecules by $B$ cells could be induced, in the absence of DCs, by exogenous retinoic acid. Therefore, only DCs from the GALT, through the secretion of retinoic acid, induce gut-tropic $B$ cells.

The authors then examined the ability of these $B$ cells to produce lgA. Only Peyer's patch-derived DCs induced de novo class switching and secretion of $\lg A$ by $B$ cells. Blocking of the retinoic acid receptor with the receptor antagonist LE540 decreased IgA secretion in these co-cultures. However, retinoic acid alone was not sufficient to induce $\lg A$ secretion, indicating that, although retinoic acid is involved, additional factors are required. Blocking of IL- 6 or IL-5 (cytokines that are known to induce $\lg$ A responses) with specific antibodies, together with LE540 (but not on their own), lead to a reduction in $\lg A$ secretion.

So, these data show that GALT-derived DCs directly induce gut tropism in a retinoic-acid-dependent manner, and they induce class switching to $\lg \mathrm{A}$ in $\mathrm{B}$ cells through a synergistic mechanism that also involves retinoic acid. This study is of clinical relevance as it might explain why vitamin A deficiency exacerbates diarrhoeal disease in malnourished children.

Olive Leavy

ORIGINAL RESEARCH PAPER Mora, J. R. et al. Generation of gut-homing IgA-secreting B cells by intestinal dendritic cells. Science 314, 1157-1160 (2006)

\section{A stimulating collaboration}

The catalogue of molecules that are involved in activating or inhibiting T-cell function is ever increasing, and now another can be added to it. In a study published in The Journal of Immunology, NGFI-A-binding protein 2 (NAB2) is shown to function as a co-activator of T-cell function by promoting the transcription of interleukin-2 (IL2) mediated by the transcription factor early growth response 1 (EGR1).

NAB2 has no DNA-binding domain so it must collaborate gene transcription. In this capacity, it has been shown to be both a coactivator and a co-repressor of EGRmediated transcription. In T cells, expression of the EGR proteins EGR1, EGR2 and EGR3 is markedly upregulated after ligation of the T-cell with transcription factors to affect receptor (TCR). EGR1 is known to stimulate T-cell activation and promote IL-2 production, whereas EGR2 and EGR3 have inhibitory effects on T cells. So, how does NAB2 influence EGR-mediated transcription in $\mathrm{T}$ cells?

Initial analysis of NAB2 expression revealed that the highest levels of NAB2 protein expression coincided with maximum IL2 transcription in T cells stimulated with CD3- and CD28-specific antibodies. This led the authors to suggest that NAB2 might have an activating role in $\mathrm{T}$ cells. Consistent with this hypothesis, overexpression of NAB2 increased the transcription of a reporter gene driven by the IL2 promoter. By contrast, knockdown of NAB2 expression using small interfering RNA led to a
$\Rightarrow$ DENDRITIC CELLS

\section{Terminal differentiation of plasmacytoid dendritic cells}

Plasmacytoid dendritic cells (pDCs) share several features with conventional dendritic cells (cDCs) but they are unique in their ability to produce large amounts of type I interferons in response to viral infection. However, the signals that drive pDC differentiation are not clearly understood. Now, the laboratory of Susan Chan and Philippe Kastner reports in Blood that the zinc-finger transcription factor Ikaros is required for the generation of peripheral pDCs from a committed pDC population in the bone marrow.
Ikaros is essential for the development of multiple haematopoietic lineages. Previous studies have shown that expression of a dominant-negative form of lkaros resulted in the lack of both $C D 8 \alpha^{+}$and $C D 8 \alpha^{-}$subsets of cDCs. By contrast, an lkaros null mutation resulted in selective loss of the $\mathrm{CD} 8 \alpha^{-}$subset of $\mathrm{cDC}$ s. In this study, Chan and colleagues studied mice expressing a hypomorphic mutant of Ikaros (Ik $\left.\mathrm{k}^{\mathrm{L} / L}\right)$ which resulted in lowlevel expression of lkaros protein in haematopoietic cells. Using the

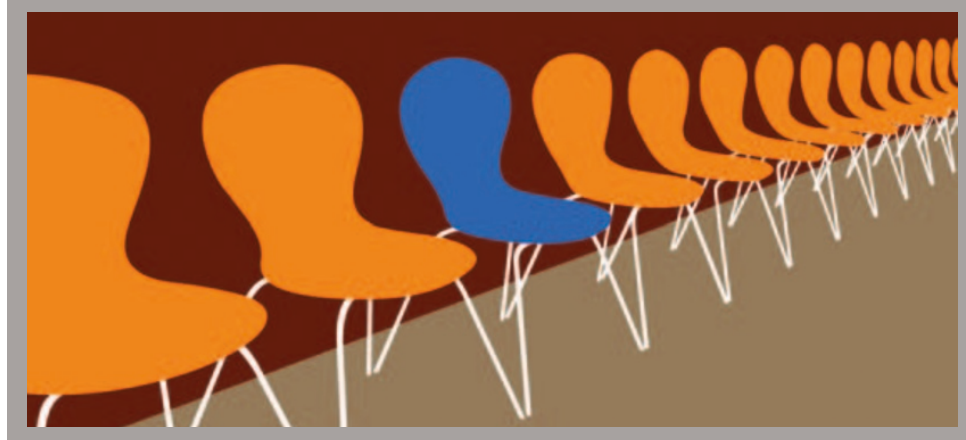

